\title{
Berge cycles in non-uniform hypergraphs
}

\author{
Zoltán Füredi* \\ Alfréd Rényi Institute of Mathematics \\ Budapest, Hungary \\ z-furedi@illinois.edu \\ Alexandr Kostochka ${ }^{\dagger}$ \\ Department of Mathematics \\ University of Illinois at Urbana-Champaign \\ Urbana, IL, U.S.A. \\ kostochk@math.uiuc.edu \\ Ruth Luo \\ Department of Mathematics \\ University of California, San Diego \\ La Jolla, CA, U.S.A. \\ ruluo@ucsd.edu
}

Submitted: Feb 4, 2020; Accepted: Jul 3, 2020; Published: Jul 24, 2020

(C) The authors. Released under the CC BY-ND license (International 4.0).

\begin{abstract}
We consider two extremal problems for set systems without long Berge cycles. First we give Dirac-type minimum degree conditions that force long Berge cycles. Next we give an upper bound for the number of hyperedges in a hypergraph with bounded circumference. Both results are best possible in infinitely many cases.
\end{abstract}

Mathematics Subject Classifications: 05C65, 05C35, 05C38

\section{Introduction}

\subsection{Classical results on longest cycles in graphs}

The circumference $c(G)$ of a graph $G$ is the length of its longest cycle. In particular, if a graph has a cycle $C$ which covers all of its vertices, $V(C)=V(G)$, we say it is hamiltonian. A classical result of Dirac states that high minimum degree in a graph forces hamiltonicity.

\footnotetext{
${ }^{*}$ Research supported in part by the Hungarian National Research, Development and Innovation Office NKFIH grant KH-130371.

${ }^{\dagger}$ Research is supported in part by NSF grant DMS-1600592 and grants 18-01-00353A and 19-01-00682 of the Russian Foundation for Basic Research.

$\ddagger$ University of California, San Diego, La Jolla, CA 92093, USA.
} 
Theorem 1 (Dirac [4]). Let $n \geqslant 3$, and let $G$ be an $n$-vertex graph with minimum degree $\delta(G)$. If $\delta(G) \geqslant n / 2$, then $G$ contains a hamiltonian cycle. If $G$ is 2-connected, then $c(G) \geqslant \min \{n, 2 \delta(G)\}$.

Inspired by this theorem, it is common in extremal combinatorics to refer to results in which a minimum degree condition forces some structure as a Dirac-type condition. The second part of Theorem 1 cannot be extended to non 2-connected graphs: let $\mathcal{F}_{n, k}$ be the family of graphs in which each block (inclusion maximal 2-connected subgraph) of the graph is a copy of $K_{k-1}$. Every $F \in \mathcal{F}_{n, k}$ has minimum degree $k-2$, but its longest cycle has length $k-1$.

Theorem 2 (Erdős, Gallai [6]). Let $G$ be an n-vertex graph with no cycle of length $k$ or longer. Then $e(G) \leqslant \frac{n-1}{k-2}\left(\begin{array}{c}k-1 \\ 2\end{array}\right)$.

So the graphs in $\mathcal{F}_{n, k}$ have the maximum number of edges among the $n$-vertex graphs with circumference $k-1$. They also maximize the number of cliques of any size:

Theorem 3 (Luo [12]). Let $G$ be an n-vertex graph with no cycle of length $k$ or longer. Then the number of copies of $K_{r}$ in $G$ is at most $\frac{n-1}{k-2}\left(\begin{array}{c}k-1 \\ r\end{array}\right)$.

\subsection{Known results on cycles in hypergraphs}

A hypergraph $\mathcal{H}$ is a set system. We often refer to the ground set as the set of vertices $V(\mathcal{H})$ of $\mathcal{H}$ and to the sets as the hyperedges $E(\mathcal{H})$ of $\mathcal{H}$. When there is no ambiguity, we may also refer to the hyperedges as edges. In this paper, we prove versions of Theorems 1 and 2 for hypergraphs with no restriction on edge sizes. Namely, we seek long Berge cycles.

A Berge cycle of length $\ell$ in a hypergraph is a set of $\ell$ distinct vertices $\left\{v_{1}, \ldots, v_{\ell}\right\}$ and $\ell$ distinct edges $\left\{e_{1}, \ldots, e_{\ell}\right\}$ such that $\left\{v_{i}, v_{i+1}\right\} \subseteq e_{i}$ with indices taken modulo $\ell$. The vertices $\left\{v_{1}, \ldots, v_{\ell}\right\}$ are called representative vertices of the Berge cycle.

A Berge path of length $\ell$ in a hypergraph is a set of $\ell+1$ distinct vertices $\left\{v_{1}, \ldots, v_{\ell+1}\right\}$ and $\ell$ distinct edges $\left\{e_{1}, \ldots, e_{\ell}\right\}$ such that $\left\{v_{i}, v_{i+1}\right\} \subseteq e_{i}$ for all $1 \leqslant i \leqslant \ell$. The vertices $\left\{v_{1}, \ldots, v_{\ell+1}\right\}$ are called representative vertices of the Berge path.

For a hypergraph $\mathcal{H}$, the 2 -shadow of $\mathcal{H}$, denoted $\partial_{2} \mathcal{H}$, is the graph on the same vertex set such that $x y \in E\left(\partial_{2} \mathcal{H}\right)$ if and only if $\{x, y\}$ is contained in an edge of $\mathcal{H}$.

Note that if we require no conditions on multiplicities of edges, then we can arbitrarily add edges of size 1 without creating new Berge cycles or Berge paths. From now on, we only consider simple hypergraphs, i.e., those without multiple edges (except if it is stated otherwise).

Bermond, Germa, Heydemann, and Sotteau [1] were among the first to prove Diractype results for uniform hypergraphs without long Berge cycles: Let $k>r$ and $\mathcal{H}$ be an $r$-uniform hypergraph with minimum degree $\delta(\mathcal{H}) \geqslant\left(\begin{array}{c}k-2 \\ r-1\end{array}\right)+(r-1)$, then $\mathcal{H}$ contains a Berge cycle of length at least $k$. For large $n$, generalizations and results for linear hypergraphs are proved by Jiang and Ma [9]. Coulson and Perarnau [3] proved that if $\mathcal{H}$ is an $r$-uniform hypergraph on $n$ vertices, $r=o(\sqrt{n})$, and $\mathcal{H}$ has minimum degree 
$\delta(\mathcal{H})>\left(\begin{array}{c}\lfloor(n-1) / 2\rfloor \\ r-1\end{array}\right)$, then $\mathcal{H}$ contains a Berge hamiltonian cycle. Ma, Hou, and Gao [13, 14] studied $r$-uniform hypergraphs and improved the result of Bermond, et al. for hamiltonian Berge cycles: if $n \geqslant 2 r+4$ and $\delta(\mathcal{H})>\left(\begin{array}{c}\lfloor(n-1) / 2\rfloor \\ r-1\end{array}\right)+\lceil(n-1) / 2\rceil$, then $\mathcal{H}$ has a Hamiltonian Berge cycle. Note that this also covers some small cases of $n$ left open by Coulson and Perarnau.

Our new results differ from these in several aspects. We consider non-uniform hypergraphs, prove exact formulas, prove results for every $n$ (or every $n>14$ ), and use only classical tools mentioned above and in Section 3.1.

\section{New results}

Our first result is a Dirac-type condition that forces hamiltonian Berge cycles.

Theorem 4. Let $n \geqslant 15$ and let $\mathcal{H}$ be an $n$-vertex hypergraph such that $\delta(\mathcal{H}) \geqslant 2^{(n-1) / 2}+1$ if $n$ is odd, or $\delta(\mathcal{H}) \geqslant 2^{n / 2-1}+2$ if $n$ is even. Then $\mathcal{H}$ contains a Berge hamiltonian cycle.

The following four constructions show that the bounds in Theorem 4 cannot be decreased for any $n$.

- Let $n$ be odd. Let $\mathcal{H}$ be the $n$-vertex hypergraph on the ground set $[n]$ with edges $\{A: A \subseteq[(n+1) / 2]\} \cup\{B: B \subseteq\{(n+1) / 2, \ldots n\}\}$. Then $\delta(\mathcal{H})=2^{(n-1) / 2}$ and $\mathcal{H}$ has no hamiltonian Berge cycle (because it has a cut vertex).

- Let $n$ be even. Let $\mathcal{H}$ be the $n$-vertex hypergraph on the ground set $[n]$ with edges $\{A: A \subseteq[n / 2]\} \cup\left\{B: B \subseteq\{(n / 2+1, \ldots n\}\}\right.$ and the set $[n]$. Then $\delta(\mathcal{H})=2^{n / 2-1}+1$ and $\mathcal{H}$ has no hamiltonian Berge cycle (because it has a cut edge, $[n]$ ).

- Let $n$ be odd. Let $\mathcal{H}$ be the $n$-vertex hypergraph on the ground set $[n]$ obtained by taking all edges with at most one vertex in $[(n+1) / 2]$. Then $\delta(\mathcal{H})=2^{(n-1) / 2}$, and $\mathcal{H}$ cannot contain a Berge cycle with two consecutive representative vertices in $[(n+1) / 2]$.

- Let $n$ be even. Let $\mathcal{H}$ be the $n$-vertex hypergraph on the ground set $[n]$ obtained by taking all edges with at most one vertex in $[n / 2+1]$ and the edge $[n]$. Then $\delta(\mathcal{H})=2^{n / 2-1}+1$, and $\mathcal{H}$ cannot contain a Berge cycle with two instances of two consecutive representative vertices in $[n / 2+1]$ (because only one edge of $\mathcal{H}$ contains multiple vertices in $[n / 2+1])$.

Next, we consider hypergraphs without long Berge paths or cycles.

Theorem 5. Let $k \geqslant 2$ and let $\mathcal{H}$ be a hypergraph such that $\delta(\mathcal{H}) \geqslant 2^{k-2}+1$. Then $\mathcal{H}$ contains a Berge path with $k$ base vertices.

A vertex disjoint union of complete hypergraphs of $k-1$ vertices shows that this bound is best possible for $n:=|V(\mathcal{H})|$ divisible by $(k-1)$.

We note that Ma, et al. [14] also proved Dirac-type bounds for the existence of long Berge paths in $r$-uniform hypergraphs, but their results do not imply Theorem 5 .

Theorem 6. Let $k \geqslant 3$ and let $\mathcal{H}$ be a hypergraph such that $\delta(\mathcal{H}) \geqslant 2^{k-2}+2$. Then $\mathcal{H}$ contains a Berge cycle of length at least $k$. 
The following constructions show that the bound in Theorem 6 is best possible when $n$ is divisible by $(k-1)$ and also when $n \equiv 1 \bmod (k-1)$ for $n>(k-1)\left(2^{k-2}+1\right)$. In the first case, take a vertex disjoint union of complete hypergraphs with $k-1$ vertices and add one more set, namely $[n]$. In the other case, take $m:=(n-1) /(k-1) \geqslant 2^{k-2}+1$ disjoint $(k-1)$-sets $A_{1}, \ldots, A_{m}$ and an element $x$ such that $[n]=\left(\cup_{1 \leqslant i \leqslant m} A_{i}\right) \cup\{x\}$. Then define $\mathcal{H}$ as the union of complete hypergraphs on the sets $A_{i}$ 's together with the edges of the form $A_{i} \cup\{x\}$. If we do not insist on connectedness, then $\left(2^{k-2}+1\right)$-regular examples can be constructed for all $n \geqslant k^{2} 2^{k-2}$.

Finally, we prove a hypergraph version of Theorem 2 .

Theorem 7. Let $n \geqslant k \geqslant 3$ and let $\mathcal{H}$ be an n-vertex hypergraph with no Berge cycle of length $k$ or longer. Then

$$
e(\mathcal{H}) \leqslant 2+\frac{n-1}{k-2}\left(2^{k-1}-2\right) .
$$

The bound in Theorem 7 is best possible when $n \equiv 1 \bmod (k-2)$. Take $m:=$ $(n-1) /(k-2)$ and disjoint sets $A_{1}, \ldots, A_{m}$ of size $k-2$. Let $x$ be a new element, and set $[n]=\left(\cup_{1 \leqslant i \leqslant m} A_{i}\right) \cup\{x\}$. Define $\mathcal{H}$ to be the union of all sets $A$ such that there exists an $i$ with $A \backslash\{x\} \subseteq A_{i}$. Note that the 2 -shadow $\partial_{2}(\mathcal{H})$ is in the family $\mathcal{F}_{n, k}$ defined before Theorem 2.

It would be interesting to find $\max \delta(\mathcal{H})$ for Theorems 5 and 6 for other values of $n$, and also for the cases when $\mathcal{H}$ is connected or 2-connected respectively. Moreover we also ask to improve the bound for Theorem 7 in the case where $\mathcal{H}$ is 2 -connected.

There are many exact results concerning the maximum size of uniform hypergraphs avoiding Berge paths and cycles, see the recent results of Ergemlidze et al. [7] or one by the present authors [8].

\section{Dirac type conditions for hamiltonian hypergraphs}

In this section, we present a proof for Theorem 4. The proof method relies on reducing the hypergraph to a dense nonhamiltonian graph. In the next three subsections we collect some results about such graphs. Subsections 3.4 and 3.5 contain the proof for hypergraphs.

\subsection{Classical tools}

Let $G$ be an $n$-vertex graph. The hamilton-closure of $G$ is the unique graph $C(G)$ of order $n$ that can be obtained from $G$ by recursively joining nonadjacent vertices with degree-sum at least $n$.

Theorem 8 (Bondy, Chvátal [2]). If $C(G)$ is hamiltonian, then so is $G$.

A graph $G$ is called hamiltonian-connected if for any pair of vertices $x, y \in V(G)$ there is a hamiltonian $(x, y)$-path. The following corollary can be obtained from Theorem 8 or from the classical result of Pósa [15]: If for every pair of nonadjacent vertices $x, y \in V(G)$ we have $d(x)+d(y) \geqslant|V(G)|+1$, then $G$ is hamiltonian-connected. 
Corollary 9. If $e(G) \geqslant\left(\begin{array}{l}n \\ 2\end{array}\right)-2$ and $n \geqslant 5$ then $G$ is hamiltonian-connected.

We will need the following result about the structure of matchings in bipartite graphs. It is a well known fact in the theory of transversal matroids (but one can also give a short, direct proof finding an $M_{3} \subseteq M_{1} \cup M_{2}$ ).

Theorem 10. Let $G[X, Y]$ be a bipartite graph. Suppose that there is a matching $M_{1}$ in $G$ joining the vertices of $X_{1} \subseteq X$ and $Y_{1} \subseteq Y$. Suppose also that we have another matching $M_{2}$ with end vertices $X_{2} \subseteq X$ and $Y_{2} \subseteq Y$ such that $Y_{2} \subseteq Y_{1}$. Then there exists a third matching $M_{3}$ from $X_{3} \subseteq X$ to $Y_{3} \subseteq Y$ such that

$$
Y_{3}=Y_{1} \quad \text { and } \quad X_{3} \supseteq X_{2} .
$$

Theorem 11 (Erdös [5]). Let $n, d$ be integers with $1 \leqslant d \leqslant\left\lfloor\frac{n-1}{2}\right\rfloor$, and $\operatorname{set} h(n, d):=$ $\left(\begin{array}{c}n-d \\ 2\end{array}\right)+d^{2}$. If $G$ is a nonhamiltonian graph on $n$ vertices with minimum degree $\delta(G) \geqslant d$, then

$$
e(G) \leqslant \max \left\{h(n, d), h\left(n,\left\lfloor\frac{n-1}{2}\right\rfloor\right)\right\}=: e(n, d) .
$$

\subsection{A lemma for nonhamiltonian graphs}

The lemma below follows from a result of Voss [16] (and from the even more detailed descriptions by Jung [10] and Jung, Nara [11]). We only state and use a weaker version and for completeness include a short proof. Define five classes of nonhamiltonian graphs.

Let $n=2 k+2, V=V_{1} \cup V_{2},\left|V_{1}\right|=\left|V_{2}\right|=k+1,\left(V_{1} \cap V_{2}=\varnothing\right)$. We say that $G \in \mathcal{G}_{1}$ if its edge set is the union of two complete graphs with vertex sets $V_{1}$ and $V_{2}$ and it contains at most one further edge $e_{0}$ (joining $V_{1}$ and $V_{2}$ );

- Let $n=2 k+1, V=V_{1} \cup V_{2},\left|V_{1}\right|=\left|V_{2}\right|=k+1, V_{1} \cap V_{2}=\left\{x_{0}\right\}$. We say that $G \in \mathcal{G}_{2}$ if its edge set is the union of two complete graphs with vertex sets $V_{1}$ and $V_{2}$;

- Let $n=2 k+2, V=V_{1} \cup V_{2},\left|V_{1}\right|=k+1,\left|V_{2}\right|=k+2, V_{1} \cap V_{2}=\left\{x_{0}\right\}$. We say that $G \in \mathcal{G}_{3}$ if its edge set is the union of a complete graph with vertex set $V_{1}$ and a 2-connected graph $G_{2}$ with vertex set $V_{2}$ such that $\operatorname{deg}_{G}(v) \geqslant k$ for every vertex $v \in V$;

- Let $n=2 k+1, V=V_{1} \cup V_{2},\left|V_{1}\right|=k,\left|V_{2}\right|=k+1,\left(V_{1} \cap V_{2}=\varnothing\right)$. We say that $G \in \mathcal{G}_{4}$ if $V_{2}$ is an independent set, and its edge set contains all edges joining $V_{1}$ and $V_{2}$;

- Let $n=2 k+2, V=V_{1} \cup V_{2},\left|V_{1}\right|=k,\left|V_{2}\right|=k+2,\left(V_{1} \cap V_{2}=\varnothing\right)$. We say that $G \in \mathcal{G}_{5}$ if $V_{2}$ contains at most one edge $e_{0}$ and $\operatorname{deg}_{G}(v) \geqslant k$ for every vertex $v \in V$ (so its edge set contains all but at most two edges joining $V_{1}$ and $V_{2}$ ).

Lemma 12. Let $k \geqslant 3$ be an integer, $n \in\{2 k+1,2 k+2\}$. Suppose that $G$ is an n-vertex nonhamiltonian graph with $\delta(G) \geqslant k=\lfloor(n-1) / 2\rfloor, V:=V(G)$. Then $G \in \mathcal{G}_{1} \cup \cdots \cup \mathcal{G}_{5}$.

Proof. Suppose first that $G$ is not 2-connected. Then there exist two blocks $B_{1}, B_{2}$ of $G$ (i.e., $B_{i}$ is a maximal 2-connected subgraph or a $K_{2}$ ) which are endblocks, i.e., for $i=1,2$ there is a vertex $v_{i} \in B_{i}$ such that $V\left(B_{i}\right) \backslash\left\{v_{i}\right\}$ does not meet any other block. Then $\{v\} \cup N(v) \subset V\left(B_{i}\right)$ for all $v \in V\left(B_{i}\right) \backslash\left\{v_{i}\right\}$, so an endblock has at least $k+1$ vertices and 
if $\left|V\left(B_{i}\right)\right|=k+1$ then it is a clique. If $B_{1}$ and $B_{2}$ are disjoint then we get $n=2 k+2$, and $G \in \mathcal{G}_{1}$. If $B_{1}$ and $B_{2}$ meet, then $G$ has no other blocks, and $G \in \mathcal{G}_{2} \cup \mathcal{G}_{3}$.

Suppose now that $G$ is 2-connected. By the second part of Dirac's theorem (Theorem 1), the length of a longest cycle $C$ of $G$ is at least $2 k$. If $|V(C)|=n-1$, assume $C=v_{1} \ldots v_{n-1} v_{1}$ and $v_{n} \notin V(C)$. Then $v_{n}$ has at least $k$ neighbors in $C$, with no two of them appearing consecutively (otherwise we could extend $C$ to a hamiltonian cycle). Without loss of generality, let $N\left(v_{n}\right)=\left\{v_{1}, v_{3}, \ldots, v_{2 k-1}\right\}$. If for some $i<j$ such that $v_{i}, v_{j} \in N\left(v_{n}\right), v_{i+1} v_{j+1} \in E(G)$, then we obtain the hamiltonian cycle $v_{1} v_{2} \ldots v_{i} v_{n} v_{j} v_{j-1} \ldots v_{i+1} v_{j+1} v_{j+2} \ldots v_{n-1} v_{1}$. Therefore the vertices in $C$ of even parity, together with $v_{n}$, form an independent set. In case of $n=2 k+1$ we get $G \in \mathcal{G}_{4}$. If $n=2 k+2$ then in the same way we get that $\left\{v_{2 k+1}\right\} \cup\left\{v_{2}, v_{4}, \ldots, v_{2 k-2}\right\}$ together with $v_{n}$ is also independent, so the set $\left\{v_{2}, \ldots, v_{2 k-2}\right\} \cup\left\{v_{2 k}, v_{2 k+1}, v_{n}\right\}$ contains only the edge $v_{2 k} v_{2 k+1}, G \in \mathcal{G}_{5}$.

Finally, consider the case that $|V(C)|=n-2$, (i.e., $n=2 k+2$ ) and let $x, y \notin V(C)$. We claim that $x y \notin E(G)$. Indeed, suppose to the contrary, that $x y \in E(G)$. Without loss of generality, $A:=\left\{v_{1}, v_{3}, \ldots, v_{2 k-3}\right\} \subseteq N(x)$ or $\left.\left(A \backslash\left\{v_{2 k-3}\right\}\right) \cup\left\{v_{2 k-2}\right\}\right) \subseteq N(x)$. Note that for any $v_{i} \in N(x),\left\{v_{i-2}, v_{i-1}, v_{i+1}, v_{i+2}\right\} \cap N(y)=\varnothing$ (indices are taken modulo $2 k$ ), because we can remove a segment of $C$ with at most 3 vertices and replace it with a segment with at least 4 vertices containing the edge $x y$. This leads to a contradiction because there is not enough room on the $2 k$-cycle $C$ to distribute the at least $k-1$ vertices of $N(y)-\{x\}$.

If $x y \notin E(G)$ then without loss of generality let $N(x)=\left\{v_{1}, v_{3}, \ldots v_{2 k-1}\right\}$. Then the set $\{x\} \cup\left\{v_{2}, \ldots, v_{2 k}\right\}$ is an independent set. If $y v_{i} \in E(G)$ for some $i \in\{2,4, \ldots, 2 k\}$, then because $y$ has $k$ neighbors in $C$ and no two of them appear consecutively, $N(y)=$ $\left\{v_{2}, v_{4}, \ldots, v_{2 k}\right\}$, and we obtain a hamiltonian cycle by replacing the segment $v_{1} v_{2} v_{3} v_{4}$ of $C$ with the path $v_{1} x v_{3} v_{2} y v_{4}$. Therefore $V_{2}:=\left\{v_{2}, v_{4}, \ldots, v_{2 k}\right\} \cup\{x, y\}$ is an independent set of size $k+2$, and so $G \in \mathcal{G}_{5}$.

\subsection{A maximality property of the graphs in $\mathcal{G}_{1} \cup \ldots \cup \mathcal{G}_{5}$}

Let $G \in \mathcal{G}_{1} \cup \cdots \cup \mathcal{G}_{5}$ be a graph. Delete a set of edges $\mathcal{A}$ from $E(G)$ where $|\mathcal{A}| \leqslant 1$ for $G \in \mathcal{G}_{2} \cup \mathcal{G}_{3} \cup \mathcal{G}_{4}$ and $|\mathcal{A}| \leqslant 2$ for $G \in \mathcal{G}_{1} \cup \mathcal{G}_{5}$. Then add a set of new edges $\mathcal{B}$ as defined below:

- For $G \in \mathcal{G}_{1},|\mathcal{B}|=2$ and it consists of any two disjoint pairs joining $V_{1}$ and $V_{2}$;

- for $G \in \mathcal{G}_{2} \cup \mathcal{G}_{3},|\mathcal{B}|=1$ and it consists of any pair $x_{1} x_{2}$ joining $V_{1} \backslash\left\{x_{0}\right\}$ and $V_{2} \backslash\left\{x_{0}\right\}$ (here $x_{1} \in V_{1}$ and $x_{2} \in V_{2}$ );

- for $G \in \mathcal{G}_{4},|\mathcal{B}|=1$ and it consists of any pair contained in $V_{2}$;

— and for $G \in \mathcal{G}_{5},|\mathcal{B}|=2$ and it consists of any two distinct pairs contained in $V_{2}$.

Lemma 13. If $k \geqslant 6$, then the graph $(E(G) \backslash \mathcal{A}) \cup \mathcal{B}$ defined by the above process is hamiltonian, except if $G \in \mathcal{G}_{3}, x_{0}$ has exactly two neighbors $x_{2}$ and $y_{2}$ in $V_{2}, \mathcal{A}=\left\{x_{0} y_{2}\right\}$, $\mathcal{B}=\left\{x_{1} x_{2}\right\}$, and $G\left[V_{2} \backslash\left\{x_{0}\right\}\right]$ is either a $K_{k+1}$ or misses only the edge $x_{2} y_{2}$.

Proof. If $G \in \mathcal{G}_{1}$ and we add two disjoint edges $x_{1} x_{2}$ and $y_{1} y_{2}$ joining $V_{1}$ and $V_{2}\left(x_{1}, y_{1} \in\right.$ $\left.V_{1}\right)$ then to form a hamiltonian cycle we need an $x_{1}, y_{1}$ path $P_{1}$, and a $x_{2}, y_{2}$ path $P_{2}$ of 
length $k, V\left(P_{i}\right)=V_{i}$ and $E\left(P_{i}\right) \subset E(G) \backslash \mathcal{A}$. Such paths exist because the graph $G\left[V_{i}\right] \backslash \mathcal{A}$ has at least $\left(\begin{array}{c}k+1 \\ 2\end{array}\right)-2$ edges, so it satisfies the condition of Corollary 9 .

If $G \in \mathcal{G}_{2} \cup \mathcal{G}_{3}$ and we add an edge $x_{1} x_{2}$ joining $V_{1} \backslash\left\{x_{0}\right\}$ and $V_{2} \backslash\left\{x_{0}\right\}$ then we need paths $P_{1}, P_{2}$ of length $\left|V_{i}\right|-1$ joining $x_{i}$ to $x_{0}, V\left(P_{i}\right)=V_{i}$ and $E\left(P_{i}\right) \subset E(G) \backslash \mathcal{A}$. If $G\left[V_{i}\right] \backslash \mathcal{A}$ satisfies the condition of Corollary 9 then we can find $P_{i}$. The only missing case is when $\left|V_{2}\right|=k+2$ (so $G \in \mathcal{G}_{3}$ ). Let $G_{2}$ be the graph on $\left|V_{2}\right|+1$ vertices obtained from $G\left[V_{2}\right] \backslash \mathcal{A}$ by adding a new vertex $x_{2}^{\prime}$ and two edges $x_{0} x_{2}^{\prime}$ and $x_{2} x_{2}^{\prime}$. If $G_{2}$ has a hamiltonian cycle $C$ then it should contain $x_{0} x_{2}^{\prime}$ and $x_{2} x_{2}^{\prime}$ so the rest of the edges of $C$ can serve as $P_{2}$ we are looking for. Consider the hamilton-closure $C\left(G_{2}\right)$ and apply Theorem 8 to $G_{2}$. Since the degrees of $V_{2} \backslash\left\{x_{0}\right\}$ in $G_{2}$ are at least $k-1$ and $2(k-1) \geqslant k+3=\left|V\left(G_{2}\right)\right|, C\left(G_{2}\right)$ is a complete graph on $V_{2} \backslash\left\{x_{0}\right\}$. So $C\left(G_{2}\right)$ is hamiltonian unless the only neighbors of $x_{0}$ in $G_{2}$ are $x_{2}$ and $x_{2}^{\prime}$. Hence $N_{G}\left(x_{0}\right) \cap V_{2}=\left\{x_{2}, y_{2}\right\}$ and $\mathcal{A}=\left\{x_{0} y_{2}\right\}$.

The last case is when $G \in \mathcal{G}_{5},|\mathcal{A}|=2, \mathcal{B}=\left\{e_{1}, e_{2}\right\}$ (two distinct edges inside $V_{2}$ ). (The proofs of the other cases, especially when $G \in \mathcal{G}_{4}$ are easier). We create a graph $H_{0}$ from $G$ as follows: Delete the edge $e_{0}$ (if it exists), delete the edges of $\mathcal{A}$ joining $V_{1}$ and $V_{2}$, add two new vertices $z_{1}, z_{2}$ to $V_{1}$ and join $z_{i}$ to the endpoints of $e_{i}$. We obtain the graph $H$ by adding all possible $\left(\begin{array}{c}k+2 \\ 2\end{array}\right)$ pairs from $V_{1} \cup\left\{z_{1}, z_{2}\right\}$ to $H_{0}$.

If $H$ is hamiltonian then its hamiltonian cycle must use only edges of $H_{0}$ (because $V_{2}$ is an independent set of size $k+2$ in $H$ ). If the graph $H_{0}$ is hamiltonian then its hamiltonian cycle must use the two edges of the degree 2 vertex $z_{i}$, so $\left(G \backslash\left(\left\{e_{0}\right\} \cup \mathcal{A}\right)\right) \cup \mathcal{B}$ is hamiltonian as well. So it is sufficient to show that $H$ has a hamiltonian cycle.

Let $A$ be the graph on $V(H)$ consisting of the edges of $\mathcal{A}$ joining $V_{1}$ and $V_{2}$ together with the (at most) two missing pairs $E\left(K\left(V_{1}, V_{2}\right)\right) \backslash E(G)$. We will again apply Theorem 8 to $H$, so consider the hamilton-closure $C(H)$. The degree $\operatorname{deg}_{H}(x)$ of an $x \in V_{1}$ is $(2 k+3)-\operatorname{deg}_{A}(x)$. The degree $\operatorname{deg}_{H}(y)$ of a $y \in V_{2}$ is at least $\left|V_{1}\right|-\operatorname{deg}_{A}(y)=k-\operatorname{deg}_{A}(y)$. Since $\operatorname{deg}_{A}(x)+\operatorname{deg}_{A}(y) \leqslant|E(A)|+1 \leqslant 5$ we get for $k \geqslant 6$ that

$$
\operatorname{deg}_{H}(x)+\operatorname{deg}_{H}(y) \geqslant(3 k+3)-\left(\operatorname{deg}_{A}(x)+\operatorname{deg}_{A}(y)\right) \geqslant 3 k-2 \geqslant 2 k+4=|V(H)| .
$$

So $C(H)$ contains the complete bipartite graph $K\left(V_{1}, V_{2}\right)=K_{k, k+2}$. Then it is really a simple task to find a hamiltonian cycle in $C(H)$ and therefore $(E(G) \backslash \mathcal{A}) \cup \mathcal{B}$ is hamiltonian.

\subsection{Proof of Theorem 4, reducing the hypergraph to a dense graph}

Fix $\mathcal{H}$ to be an $n$ vertex hypergraph satisfying the minimum degree condition. We will find a hamiltonian Berge cycle in $\mathcal{H}$.

Recall that $H=\partial_{2}(\mathcal{H})$ denotes the 2-shadow of $\mathcal{H}$, a graph on $V=V(\mathcal{H})$. Define a bipartite graph $B:=B[E(\mathcal{H}), E(H)]$ with parts $E(\mathcal{H})$ and $E(H)$ and with edges $\{h, x y\}$ where a hyperedge $h \in E(\mathcal{H})$ is joined to the graph edge $x y \in E(H)$ if $\{x, y\} \subseteq h$. In the case of $\{x, y\} \in \mathcal{H}$ we consider the edge $x y \in E(H)$ and $\{x, y\} \in E(\mathcal{H})$ as two distinct objects of $B$, so $B$ is indeed a bipartite graph (with $|E(\mathcal{H})|+|E(H)|$ vertices and no loops). Let $M$ be a maximum matching of $B$. So $M$ can be considered as a partial injection of maximum size, i.e., a bijection $\phi$ between two subsets $\mathcal{M} \subseteq E(\mathcal{H})$ and $\mathcal{E} \subseteq E(H)$ such 
that $|\mathcal{M}|=|\mathcal{E}|, \phi(m) \subseteq m$ for $m \in \mathcal{M}$ (and $\phi\left(m_{1}\right) \neq \phi\left(m_{2}\right)$ for $\left.m_{1} \neq m_{2}\right)$. Consider the subgraph $G=(V, \mathcal{E})$ of $H$. Then $G$ does not have a hamiltonian cycle, otherwise by replacing the edges of a hamiltonian cycle with their corresponding matched hyperedges in $M$, we obtain a hamiltonian Berge cycle in $\mathcal{H}$ (with representative vertices in the same order). In this subsection we are going to prove that

$$
\delta(G) \geqslant\lfloor(n-1) / 2\rfloor:=k .
$$

Since $G$ has no hamiltonian cycle and $k \geqslant 7$, if (1) holds, then by Lemma $12, G \in$ $\mathcal{G}_{1} \cup \cdots \cup \mathcal{G}_{5}$. We will consider this case and prove the remainder of Theorem 4 in the next subsection.

Let $\mathcal{H}_{2}:=E(\mathcal{H}) \cap \partial_{2}(\mathcal{H})$, the set of 2-element edges of $\mathcal{H}$. We may assume that among all maximum sized matchings of $B$ the matching $M$ maximizes $\left|\mathcal{M} \cap \mathcal{H}_{2}\right|$.

Claim 14. $\mathcal{H}_{2} \subseteq \mathcal{M}, \partial_{2}(\mathcal{M})=E(H)$, and every $m \in E(\mathcal{H}) \backslash \mathcal{M}$ induces a complete graph in $G$.

Proof. If $m \in E(\mathcal{H})$ contains an edge $e \in E(H) \backslash E(G)$ then one can enlarge the matching $M$ by adding $\{m, e\}$ to it, if it is possible. Since $M$ is maximal, it cannot be enlarged, so $m \in \mathcal{M}$. This implies the second and the third statements. We also obtained that if $\{x, y\} \in E(\mathcal{H})$ then $x y \in E(G)$, so $\phi(m)=x y$ for some $m \in \mathcal{M}$. In case of $|m|>2$ we can replace the pair $\{m, x y\}$ by the pair $\{\{x, y\}, x y\}$ in $M$ and the new matching covers more edges from $\mathcal{H}_{2}$ than $M$ does (in the graph $B$ ). So $|m|=2$, all members of $\mathcal{H}_{2}$ must belong to $\mathcal{M}$.

To continue the proof of Theorem 4, let $d:=\delta(G), v \in V$ such that $D:=N_{G}(v)$, $|D|=d$. Since $G$ is not hamiltonian, Theorem 1 gives $d \leqslant k$. Let $\mathcal{H}_{v}=\{e \in \mathcal{H}: v \in e\}$ denote the edges of $H$ incident to $v,\left(\operatorname{deg}_{\mathcal{H}}(v)=\left|\mathcal{H}_{v}\right|\right)$, and split it into two parts, $\mathcal{H}_{v}=\mathcal{D} \cup \mathcal{L}$ where $\mathcal{D}:=\{e \in E(\mathcal{H}): v \in e \subseteq\{v\} \cup D\}$ and $\mathcal{L}:=\mathcal{H}_{v} \backslash \mathcal{D}$. Split $\mathcal{D}$ further into three parts according to the sizes of its edges, $\mathcal{D}=\mathcal{D}^{-} \cup \mathcal{D}_{2} \cup \mathcal{D}_{3}$ where $\mathcal{D}_{i}:=\{e \in \mathcal{D}:|e|=i\}$ (for $\left.i=2,3\right)$ and $\mathcal{D}^{-}:=\mathcal{D} \backslash\left(\mathcal{D}_{2} \cup \mathcal{D}_{3}\right)$. Since $\mathcal{D}$ can have at most $2^{d}$ members and we handle $\mathcal{D}_{2}$ and $\mathcal{D}_{3}$ separately we get

$$
|\mathcal{D}| \leqslant 2^{d}-d-\left(\begin{array}{l}
d \\
2
\end{array}\right)+\left|\mathcal{D}_{2}\right|+\left|\mathcal{D}_{3}\right|
$$

Recall that the matching $M$ in the bipartite graph $B$ can be considered as a bijection $\phi: \mathcal{M} \rightarrow \mathcal{E}$, where $\mathcal{M} \subseteq E(\mathcal{H})$ and $\mathcal{E} \subseteq E(H)$. Define another matching $M_{2}$ in $B$ by an injection $\phi_{2}: \mathcal{D}_{2} \cup \mathcal{D}_{3} \rightarrow E(G)$ as follows. If $m \in \mathcal{M} \cap\left(\mathcal{D}_{2} \cup \mathcal{D}_{3}\right)$ then $\phi_{2}(m):=\phi(m)$. In particular, since $\mathcal{D}_{2} \subseteq \mathcal{M}$, if $\{v, x\} \in \mathcal{D}_{2}$ then $\phi_{2}(\{v, x\})=v x$. If $m=\{v, x, y\} \in \mathcal{D}_{3} \backslash \mathcal{M}$ then let $\phi_{2}(m):=x y$. Since $\phi_{2}\left(\mathcal{D}_{2} \cup \mathcal{D}_{3}\right) \subseteq E(G)$ we can apply Theorem 10 to the matchings $M$ and $M_{2}$ in $B$ with $X_{1}:=\mathcal{M}, Y_{1}:=E(G)$, and $X_{2}:=\mathcal{D}_{2} \cup \mathcal{D}_{3}$. So there exists a subfamily $\mathcal{L}_{3} \subseteq \mathcal{H} \backslash\left(\mathcal{D}_{2} \cup \mathcal{D}_{3}\right)$ and a bijection $\phi_{3}:\left(\mathcal{D}_{2} \cup \mathcal{D}_{3} \cup \mathcal{L}_{3}\right) \rightarrow E(G)$. The matching $M^{\prime}$ defined by $\phi_{3}$ is also a largest matching of $B$. Every $m \in \mathcal{L}$ has an element 
$x \notin D$, so $v x \notin E(G)$. If $m$ is not matched in $M^{\prime}$, then we add $\{m, v x\}$ to $M^{\prime}$ to get a larger matching. Hence $m \in \mathcal{L}_{3}$. These yield

$$
|\mathcal{L}| \leqslant\left|\mathcal{L}_{3}\right|=e(G)-\left|\mathcal{D}_{2}\right|-\left|\mathcal{D}_{3}\right|
$$

Summing up (2) and (3), then using the lower bound for $\left|\mathcal{H}_{v}\right|$ and the upper bound of Theorem 11 for $e(G)$ we obtain

$$
2^{k}+1 \leqslant \operatorname{deg}_{\mathcal{H}}(v) \leqslant 2^{d}-\left(\begin{array}{c}
d+1 \\
2
\end{array}\right)+e(n, d)
$$

The inequality $2^{k}+1 \leqslant 2^{d}-\left(\begin{array}{c}d+1 \\ 2\end{array}\right)+e(n, d)$ does not hold for $n \geqslant 15$ and $d<k$, e.g., for $(n, k, d)=(16,7,6)$, the right hand side is only $64-21+85=128$. This completes the proof of $d=k$.

\subsection{Proof of Theorem 4, the end}

We may assume that $G \in \mathcal{G}_{1} \cup \cdots \cup \mathcal{G}_{5}$ by Lemma $12, \phi$ is a bijection $\phi: \mathcal{M} \rightarrow E(G)$ with $\phi(m) \subseteq m$ where $\mathcal{M} \subseteq E(\mathcal{H})$, and Claim 14 holds. Let $\mathcal{L}_{v}$ denote the set of edges $m \in \mathcal{H}$ containing an edge $v y$ of $E(H) \backslash E(G)$. Note that $\mathcal{L}_{v} \subseteq \mathcal{M}$. If $\operatorname{deg}_{G}(v)=k$, then the family $\mathcal{L}_{v}$ is non-empty, otherwise $\operatorname{deg}_{\mathcal{H}}(v) \leqslant 2^{k}$.

Call a graph $F$ with vertex set $V$ a Berge graph of $\mathcal{H}$ if $E(F) \subseteq E(H)$, and there exists a subhypergraph $\mathcal{F} \subseteq \mathcal{H}$, and a bijection $\psi: \mathcal{F} \rightarrow E(F)$ such that $\psi(m) \subseteq m$ for each $m \in \mathcal{F}$. We are looking for a Berge graph of $\mathcal{H}$ having a hamiltonian cycle. In particular, the graph $G$ is a Berge graph of $\mathcal{H}$ and it is almost hamiltonian. We will show that a slight change to $G$ yields a hamiltonian Berge graph of $\mathcal{H}$.

If $G \in \mathcal{G}_{2} \cup \mathcal{G}_{3}$ then choose any $v \in V_{1} \backslash\left\{x_{0}\right\}$ and let $m \in \mathcal{L}_{v}$. There exists an edge $v y \in(E(H) \backslash E(G))$ contained in $m$. Then $y \in V_{2} \backslash\left\{x_{0}\right\}$. The graph $(E(G) \backslash\{\phi(m)\}) \cup$ $\{v y\}$ is a Berge graph of $\mathcal{H}$ (we map $m$ to the edge $v y$ instead of $\phi(m))$. According to Lemma 13 (with $\mathcal{A}:=\{\phi(m)\}$ and $\mathcal{B}:=\{v y\}$ ) it is hamiltonian except if we run into the only exceptional case: $x_{0}$ has exactly two $G$-neighbors $x_{2}$ and $y_{2}$ in $V_{2}, v y=v x_{2}$, and $\phi(m)=x_{0} y_{2}$. In this case $m$ contains $\left\{x_{0}, v, x_{2}, y_{2}\right\}$ so it can be avoided by choosing $y:=y_{2}$ instead of $y:=x_{2}$.

If $G \in \mathcal{G}_{4}$ then we argue in a very similar way. Choose any $v \in V_{2}$ and let $m \in \mathcal{L}_{v}$ containing an edge $v y \in(E(H) \backslash E(G))$. Then $y \in V_{2}$ and the graph $(E(G) \backslash\{\phi(m)\}) \cup$ $\{v y\}$ is a Berge graph of $\mathcal{H}$ that is hamiltonian by Lemma 13 with $\mathcal{A}:=\{\phi(m)\}$ and $\mathcal{B}:=\{v y\}$. From now on we may suppose that $n=2 k+2$ so $\left|\mathcal{L}_{v}\right| \geqslant 2$ for $\operatorname{deg}_{G}(v)=k$.

If $G \in \mathcal{G}_{1}$ then define $\mathcal{M}_{1,2}$ as the members of $\mathcal{M}$ meeting both $V_{1}$ and $V_{2}$. The minimum degree condition on $\mathcal{H}$ implies that $\left|\mathcal{M}_{1,2}\right| \geqslant 2$. Since $\mathcal{M}_{1,2}$ can have at most one member of size 2 , we can choose an $m_{1},\left|m_{1}\right| \geqslant 3$. By symmetry we may suppose that $\left|m_{1} \cap V_{1}\right| \geqslant 2$ and let $x_{2} \in V_{2} \cap m_{1}$. Choose an element $y \in V_{2}, y \notin e_{0}, y \neq x_{2}$. Since $\left|\mathcal{L}_{y}\right| \geqslant 2$ we can choose an $m_{2} \in \mathcal{M}_{1,2}$ such that $m_{1} \neq m_{2}$ and $y \in m_{2}$. Take any pair $\left\{y_{1}, y\right\} \subseteq m_{2}$ with $y_{1} \in V_{1}$. Then one can choose an $x_{1} \in m_{1} \cap V_{1}$ so that $x_{1} \neq x_{2}$. So the pairs $\left\{x_{1}, x_{2}\right\} \subseteq m_{1}$ and $\left\{y_{1}, y\right\} \subseteq m_{2}$ are disjoint. Lemma 13 with $\mathcal{A}:=\left\{\phi\left(m_{1}\right), \phi\left(m_{2}\right)\right\}$ 
and $\mathcal{B}:=\left\{x_{1} x_{2}, y_{1} y\right\}$ implies that the graph $(E(G) \backslash \mathcal{A}) \cup \mathcal{B}$ is a hamiltonian Berge graph of $\mathcal{H}$.

If $G \in \mathcal{G}_{5}$ then $\left|\mathcal{L}_{v}\right| \geqslant 2$ for any $v \in V_{2} \backslash e_{0}$ and for all members $m$ of $\mathcal{L}_{v}$ we have $\left|m \cap V_{2}\right| \geqslant 2$. Fix $v \in V_{2} \backslash e_{0}$ and let $m_{1}$ be an arbitrary member of $\mathcal{L}_{v}$. Choose a pair $\left\{v, v^{\prime}\right\} \subseteq m_{1} \cap V_{2}$. Fix another vertex $u \in V_{2} \backslash\left(e_{0} \cup\left\{v, v^{\prime}\right\}\right)$ and let $m_{2}$ be an arbitrary member of $\mathcal{L}_{u}$. Choose a pair $\left\{u, u^{\prime}\right\} \subseteq m_{2} \cap V_{2}$. Then $u \notin\left\{v, v^{\prime}\right\}$ so the pairs $\left\{u, u^{\prime}\right\}$ and $\left\{v, v^{\prime}\right\}$ are distinct. Again, apply Lemma 13 with $\mathcal{A}:=\left\{\phi\left(m_{1}\right), \phi\left(m_{2}\right)\right\}$ and $\mathcal{B}:=\left\{u u^{\prime}, v v^{\prime}\right\}$. This completes the proof of Theorem 4 .

Remark 15. We can also show that all extremal examples are slight modifications of the four types of the sharpness examples described after Theorem 4.

\section{Dirac-type conditions for long Berge cycles}

In this section we prove Theorem 5 for Berge paths and Theorem 6 for Berge cycles. In fact we prove the two statements simultaneously.

Proof of Theorems 5 and 6 . Suppose that $\delta(\mathcal{H}) \geqslant 2^{k-2}+1, k \geqslant 3$ and that $\mathcal{H}$ has no Berge cycle of length $k$ or longer. We will show that it contains a Berge path of length $k-1$ (thus establishing Theorem 5) and then that $\delta(\mathcal{H})=2^{k-2}+1$ (which completes the proof of Theorem 6).

Choose a longest Berge path in $\mathcal{H}$ according the following rules. We say that a Berge path with edges $\left\{e_{1}, \ldots, e_{s}\right\}$ is better than a Berge path with edges $\left\{f_{1}, \ldots, f_{t}\right\}$ if

a) $s>t$ or

b) $s=t$ and $\sum\left|e_{i}\right|<\sum\left|f_{j}\right|$.

Consider a best Berge path $\mathcal{P}$ in $\mathcal{H}$. Let the base vertices of the path be $v_{1}, v_{2}, \ldots, v_{p}$. Let $e_{1}, \ldots, e_{p-1}$ be the edges of the path $\left(v_{i}, v_{i+1} \in e_{i}\right)$. First, we show that $p \geqslant k-1$. (In fact, $p \geqslant k$ follows but that will be proved later).

Indeed, let $\mathcal{H}^{(p)}$ be the hypergraph consisting of the edges of $\mathcal{H}$ containing $v_{p}$, contained in $\left\{v_{1}, \ldots, v_{p}\right\}$ and also the edges of the path, i.e.,

$$
E\left(\mathcal{H}^{(p)}\right):=\left\{e \in E(\mathcal{H}): v_{p} \in e \subseteq\left\{v_{1}, \ldots, v_{p}\right\}\right\} \cup\left\{e_{1}, \ldots, e_{p-1}\right\} .
$$

Then for $p \leqslant k-2$ (and $k \geqslant 3$ ) we have

$$
\left|E\left(\mathcal{H}^{(p)}\right)\right| \leqslant 2^{p-1}+(p-1) \leqslant 2^{k-2}<\delta(\mathcal{H}) \leqslant \operatorname{deg}_{\mathcal{H}}\left(v_{p}\right)
$$

So there exists an edge $f$ in $E(\mathcal{H}) \backslash E\left(\mathcal{H}^{(p)}\right)$ containing $v_{p}$. Then $e_{1}, \ldots, e_{p-1}, f$ form a Berge path longer than $\mathcal{P}$, a contradiction.

Now we have $p \geqslant k-1$, so we can define $W:=\left\{v_{1}, \ldots, v_{k-1}\right\}$. Let $\mathcal{P}_{1}$ be the subhypergraph consisting of the first $k-1$ edges of $\mathcal{P}, E\left(\mathcal{P}_{1}\right):=\left\{e_{1}, \ldots, e_{k-1}\right\}$ (if $p=k-1$ we take $\left.\mathcal{P}_{1}:=\mathcal{P}\right)$. Let $\mathcal{H}_{1}$ be the subhypergraph of $\mathcal{H}$ consisting of the edges incident to $v_{1}$.

Claim 16. Every edge $f \in E\left(\mathcal{H}_{1}\right) \backslash E\left(\mathcal{P}_{1}\right)$ is contained in $W:=\left\{v_{1}, \ldots, v_{k-1}\right\}$. 
Proof. First, we show that every edge $f \in E\left(\mathcal{H}_{1}\right) \backslash E\left(\mathcal{P}_{1}\right)$ avoids $\left\{v_{k}, \ldots, v_{p}\right\}$. Otherwise, if there exists an edge $f \in E\left(\mathcal{H}_{1}\right) \backslash E\left(\mathcal{P}_{1}\right)$ such that $f \cap\left\{v_{k}, \ldots, v_{p}\right\} \neq \varnothing$, then suppose that $v_{i}$ has the minimum index $(k \leqslant i \leqslant p)$ such that $v_{i}$ is a vertex of such an $f$. Then $e_{1}, \ldots, e_{i-1}$ and $f$ are forming a Berge cycle of length $i$, since these edges are all distinct and $v_{1}, v_{i} \in f$. Finally, suppose that there is an edge $f \in E\left(\mathcal{H}_{1}\right) \backslash E\left(\mathcal{P}_{1}\right)$ such that $v \in f, v \notin W$. Then $v \notin\left\{v_{1}, \ldots, v_{p}\right\}$ so the path $f, e_{1}, \ldots, v_{p}$ is longer than $\mathcal{P}$, a contradiction.

Let $\mathcal{K}$ be the family of all $2^{k-2}$ subsets of $W$ that contain $v_{1}$. We claim there is a one-to-one mapping $\varphi$ from $\mathcal{H}_{1} \backslash e_{k-1}$ to $\mathcal{K}$. The existence of such a $\varphi$ implies

$$
\delta(\mathcal{H}) \leqslant \operatorname{deg}_{\mathcal{H}}\left(v_{1}\right) \leqslant 2^{k-2}+1 .
$$

If an edge $e$ of $\mathcal{H}_{1}$ satisfies $e \subseteq W$, then let $\varphi(e)=e$. Otherwise, let $\mathcal{A} \subseteq \mathcal{H}_{1}$ be the set of the edges of $\mathcal{H} \backslash\left\{e_{k-1}\right\}$ that contain both $v_{1}$ and some vertex outside of $W$. By Claim 16, each $e \in \mathcal{A}$ must be some edge $e_{i}$ in $\mathcal{P}_{1}$. Hence it remains to show that all elements of $\mathcal{A}$ can be mapped to distinct elements of $\mathcal{K}$ that are not edges of $\mathcal{H}$.

Observe that if $e_{i} \in \mathcal{A}$ then $\left\{v_{i}, v_{i+1}\right\} \notin \mathcal{H}$. Otherwise, we get a better path by replacing $e_{i}$ by $\left\{v_{i}, v_{i+1}\right\}$. Also, for $1 \leqslant i \leqslant k-2, e_{i} \in \mathcal{A}$ implies $v_{1} \in e_{i}$ and $\left\{v_{i}, v_{i+1}\right\} \subset e_{i}$. Since $e_{i} \not \subset W$ we get $\left|e_{i}\right| \geqslant 4$ for $i \geqslant 2$. We also obtain that in case of $i \geqslant 3, e_{i} \in \mathcal{A}$ we have $\left\{v_{1}, v_{i}, v_{i+1}\right\} \notin \mathcal{P}$, and moreover $\left\{v_{1}, v_{i}, v_{i+1}\right\} \notin \mathcal{H}$ since otherwise we get a better path by replacing $e_{i}$ by $\left\{v_{1}, v_{i}, v_{i+1}\right\}$. For $3 \leqslant i \leqslant k-2$ (and $e_{i} \in \mathcal{A}$ ) define $\varphi\left(e_{i}\right)$ as $\left\{v_{1}, v_{i}, v_{i+1}\right\}$.

If $e_{2} \in \mathcal{A}$ and $\left\{v_{1}, v_{2}, v_{3}\right\} \notin \mathcal{H}$ then we proceed as above, $\varphi\left(e_{2}\right):=\left\{v_{1}, v_{2}, v_{3}\right\}$. Otherwise, if $e_{2} \in \mathcal{A}$ (so $\left|e_{2}\right| \geqslant 4$ ) and $\left\{v_{1}, v_{2}, v_{3}\right\} \in \mathcal{H}$ then $\left\{v_{1}, v_{2}, v_{3}\right\} \in \mathcal{P}$ too (otherwise, we get a better path by replacing $e_{2}$ by $\left\{v_{1}, v_{2}, v_{3}\right\}$ ). We get $e_{1}=\left\{v_{1}, v_{2}, v_{3}\right\}$ (and $e_{1} \subset e_{2}$ ). We claim that $\left\{v_{1}, v_{3}\right\} \notin \mathcal{H}$. Otherwise we rearrange the base vertices of the path $\mathcal{P}$ by exchanging $v_{1}$ and $v_{2}$ (and get the order $v_{2}, v_{1}, v_{3}, \ldots, v_{p}$ ) and observe that the Berge path $\left\{v_{2}, v_{1}, v_{3}\right\},\left\{v_{1}, v_{3}\right\}, e_{3}, \ldots, e_{p-1}$ is better than $\mathcal{P}$, a contradiction. So in this case $\varphi\left(e_{2}\right):=\left\{v_{1}, v_{3}\right\}$. Finally, if $e_{1} \in \mathcal{A}$ then $\varphi\left(e_{1}\right):=\left\{v_{1}, v_{2}\right\}$, and the definition of $\varphi$ is complete.

We have shown that $\operatorname{deg}_{\mathcal{H}}\left(v_{1}\right) \leqslant\left|\mathcal{H}_{1} \backslash\left\{e_{k-1}\right\}\right|+1 \leqslant 2^{k-2}+1$. Equality holds, so $v_{1} \in e_{k-1}$. In particular $e_{k-1}$ must exist, so $\mathcal{P}$ was a Berge path of length at least $k-1$.

Our method works for multihypergraphs as well. If the maximum multiplicity of an edge is $\mu$, then the corresponding necessary bounds on the minimum degrees are $\mu 2^{k-2}+1$ or $\mu 2^{k-2}+2$, respectively. Indeed, suppose that $\delta(\mathcal{F}) \geqslant \mu 2^{k-2}+1, k \geqslant 3$ and that $\mathcal{F}$ has no Berge cycle of length $k$ or longer. Let $\mathcal{H}$ be the simple hypergraph obtained from $\mathcal{F}$ by keeping one copy from the multiple edges. We have $\delta(\mathcal{H}) \geqslant 2^{k-2}+1$. Then Theorems 5 implies that $\mathcal{H}$ (and $\mathcal{F}$ as well) contain a Berge path with $k$ base vertices.

As in the proof of Theorem 6, consider a best Berge path $\mathcal{P}$ in $\mathcal{H}$ with base vertices $v_{1}, v_{2}, \ldots, v_{p}$ and edges $e_{1}, \ldots, e_{p-1}$. We have $p \geqslant k$. Then (4) gives $\operatorname{deg}_{\mathcal{H}}\left(v_{1}\right)=2^{k-2}+1$ and we get $\operatorname{deg}_{\mathcal{H}}\left(v_{1}\right)=\left|\mathcal{H}_{1} \backslash\left\{e_{k-1}\right\}\right|+1$. Since we also obtained $\left\{v_{1}, v_{k-1}, v_{k}\right\} \subset e_{k-1}$, the multiplicity of $e_{k-1}$ could not exceed 1 . So $\delta(\mathcal{F})$ could not exceed $\mu 2^{k-2}+1$. 


\section{$5 \quad$ Maximum number of edges}

Proof of Theorem 7 . Suppose that among all $n$-vertex hypergraphs with $c(\mathcal{H})<k$ and $e(\mathcal{H})$ edges our $\mathcal{H}$ is chosen so that $\sum_{e \in E(\mathcal{H})}|e|$ is minimized.

We claim that $\mathcal{H}$ is a downset, that is, for any $e \in E(\mathcal{H})$ and $e^{\prime} \subset e, e^{\prime} \in E(\mathcal{H})$. Indeed, if there exists a set $e^{\prime}$ and an edge $e$ such that $e^{\prime} \subset e$ where $e^{\prime} \notin E(\mathcal{H})$ and $e \in E(\mathcal{H})$, then the hypergraph obtained by replacing $e$ with $e^{\prime}$ also does not contain a Berge cyle of length $k$ or longer. This contradicts the choice of $\mathcal{H}$.

Let $H=\partial_{2} \mathcal{H}$ be the 2 -shadow of $\mathcal{H}$. Suppose that $H$ contains a cycle $C=v_{1} v_{2} \ldots v_{\ell} v_{1}$. Every edge $v_{i} v_{i+1}$ of $C$ is contained in a edge of $\mathcal{H}$. But since $\mathcal{H}$ is a downset, the edge $\left\{v_{i}, v_{i+1}\right\}$ is also contained in $E(\mathcal{H})$. Therefore $\mathcal{H}$ also contains a (Berge) cycle of length $\ell$. Hence the graph $H$ contains no cycles of length at least $k$.

Let $e_{r}(\mathcal{H})$ be the number of edges of $\mathcal{H}$ of size $r$. In $H$, every edge $e$ of $\mathcal{H}$ is represented by a clique of order $|e|$, and so $e_{r}(\mathcal{H})$ is at most the number of cliques of size $r$ in $H$. Since $c(H)<k$, each edge contains at most $k-1$ vertices. By Theorem 3 ,

$e(\mathcal{H})=e_{0}(\mathcal{H})+e_{1}(\mathcal{H})+\sum_{r=2}^{k-1} e_{r}(\mathcal{H}) \leqslant 1+n+\sum_{r=2}^{k-1} \frac{n-1}{k-2}\left(\begin{array}{c}k-1 \\ r\end{array}\right)=2+\frac{n-1}{k-2}\left(2^{k-1}-2\right)$.

\section{References}

[1] J.-C. Bermond, A. Germa, M.-C. Heydemann, D. Sotteau: Hypergraphes Hamiltoniens, in Problèmes combinatoires et thèorie des graphes (Colloq. Internat. CNRS, Univ. Orsay, Orsay, 1976). Colloq. Internat. CNRS, 260 (CNRS, Paris, 1978), 39-43.

[2] J. A. Bondy, V. Chvátal: A method in graph theory, Discrete Math. 15 (1976), 111-135.

[3] M. Coulson, G. Perarnau: A Rainbow Dirac's Theorem, arXiv:1809.06392, (2018).

[4] G. A. Dirac: Some theorems on abstract graphs, Proc. London Math. Soc. (3) 2 (1952), 69-81.

[5] P. Erdős: Remarks on a paper of Pósa, Magyar Tud. Akad. Mat. Kutató Int. Közl. 7 (1962), 227-229.

[6] P. Erdős, T. Gallai: On maximal paths and circuits of graphs, Acta Math. Acad. Sci. Hungar. 10 (1959), 337-356.

[7] B. Ergemlidze, E. Győri, A. Methuku, N. Salia, C. Tompkins, O. Zamora: Avoiding long Berge cycles, the missing cases $k=r+1$ and $k=r+2$, (English summary) Combin. Probab. Comput. 29 (2020), 423-435.

[8] Z. Füredi, A. Kostochka, R. Luo: Avoiding long Berge cycles, J. Combinatorial Theory, Ser. B 137 (2019), 55-64.

[9] T. Jiang, J. Ma: Cycles of given lengths in hypergraphs, J. Combinatorial Theory, Ser. B 133 (2018), 54-77. 
[10] H. A. Jung: On maximal circuits in finite graphs. Ann. Discrete Math. 3 (1978), 129-144.

[11] H. A. Jung, C. Nara: Note on 2-connected graphs with $d(u)+d(v) \geqslant n-4$, Arch. Math. (Basel) 39 (1982), 383-384.

[12] R. Luo: The maximum number of cliques in graphs without long cycles, J. Combinatorial Theory, Ser. B, 128 (2018), 219-226.

[13] Y. Ma, X. Hou, J. Gao: Minimum degree of 3-graphs without long linear paths, Discrete Math. 343 no. 9 (2020), 111949, 6 pp.

[14] Y. Ma, X. Hou, J. Gao: A Dirac-type theorem for uniform hypergraphs, arXiv:2004.05073, (2020).

[15] L. Pósa: A theorem concerning Hamilton lines, Magyar Tud. Akad. Mat. Kutató Int. Közl. 7 (1962), 225-226.

[16] H.-J. Voss: Maximal circuits and paths in graphs. Extreme cases. Combinatorics (Proc. Fifth Hungarian Colloq., Keszthely, 1976), Vol. II, Colloq. Math. Soc. János Bolyai, 18, North-Holland, Amsterdam-New York (1978), 1099-1122. 